Palabra Clave (La Plata), abril 2017, vol. 6, n², e017. ISSN 1853-9912

Universidad Nacional de La Plata.

Facultad de Humanidades y Ciencias de la Educación.

Departamento de Bibliotecología

\title{
Implementación de las RDA en Chile: pasado, presente y futuro
}

\author{
RDA implementation in Chile: past, present, and future
}

\section{Angela Quiroz Ubierna*}

* Biblioteca del Congreso Nacional de Chile, Santiago de Chile. Departamento de Producción de Recursos de Información, Chile I aquiroz@bcn.cl

\section{PALABRAS CLAVE}

Catalogación

Recursos, Descripción y Acceso

(RDA)

Biblioteca del Congreso Nacional de Chile

Reglas de Catalogación Anglo Americanas

BiBFRAME

FRBR-LRM

\section{KEYWORDS ABSTRACT}

Cataloguing

Resource, Description and Access (RDA)

Library of the National Congress of Chile

Anglo American Cataloging Rule BIBFRAME FRBR-LRM

\section{RESUMEN}

En el año 2010, al darse a conocer las nuevas reglas de catalogación "Recursos, Descripción y Acceso" a través del lanzamiento del RDA Toolkit, en las bibliotecas chilenas, especialmente en la Biblioteca Nacional de Chile y en la Biblioteca del Congreso Nacional de Chile, un grupo de bibliotecarios decidió comenzar a estudiarlas, ya que sólo se habían publicado en idioma inglés. Por lo anterior se creó el "Grupo de Interés RDA Chile" (GIRCH), en el cual participaron bibliotecarios de distintas instituciones. Este grupo comenzó a funcionar en 2010 y se realizaron distintas capacitaciones, en primer lugar para dar a conocer las RDA y los modelos conceptuales relacionados: FRBR, FRAD y FRSAD, pero además, para comenzar la aplicación de estas nuevas normas a nivel nacional. Sin embargo, con el pasar de los años, el grupo GIRCH cesó sus funciones (año 2014), con lo que el tema de la aplicación de las RDA quedó estancado a nivel país y se empezó a trabajar de manera local en cada biblioteca interesada en aplicar este estándar. Cuando apareció el RDA Toolkit, algunas bibliotecas chilenas se suscribieron a este recurso; no obstante, se siguió trabajando de manera aislada, sin estudiar las nuevas normas, ni la herramienta en conjunto, lo que llevó a un nivel diferente de uso y aplicación de las RDA en cada biblioteca que decidió implementarlas. No obstante lo anterior, en este documento se revisará la experiencia desarrollada por la Biblioteca del Congreso Nacional (BCN), la que a partir de agosto de 2012 generó un proyecto de implementación del modelo de catalogación RDA, tanto en los registros bibliográficos como en los de autoridad; asimismo, el caso del Sistema de Bibliotecas de la Universidad de Concepción, que en el año 2014 decidieron también aplicar RDA en todos sus registros bibliográficos y en 2015 en sus registros de autoridad. Además, se darán a conocer otros casos en los que se ha decidido implementar las RDA de manera paulatina, de manera híbrida (mezcla de la RCAA2 y RDA). Finalmente, se darán a conocer las actividades que se están realizando hoy en día en relación a las RDA, como es el caso de las capacitaciones ofrecidas por la Biblioteca del Congreso Nacional de Chile a bibliotecarios de otras instituciones del país, además de la participación en IFLA sobre el tema de RDA y los proyectos a futuro relacionados con la continuación de las capacitaciones y el estudio del nuevo modelo FRBR-LRM y el formato BIBFRAME

In the year 2010, when the new cataloguing rules "Resources, Description, and Access" (RDA) were announced through the RDA Toolkit launch, in Chilean libraries, especially in the National Library of Chile and in the National Congress of Chile Library, a group of librarians decided to start studying the RDAs, since they had only been published in English. Due to this fact, the "Grupo de Interest RDA Chile (GIRCH)" [Chile Interest Group on RDA] was created, in which librarians from different institutions collaborated. This group began in 2010 the study of this standard, and different trainings were provided, firstly, to understand RDA and the related conceptual models: FRBR, FRAD, and FRSAD, but also, to start with the implementation of these new standards nationwide. Nevertheless, as the years went by, the GIRCH ceased its activities (2013), bringing that the RDA implementation at country level was stagnated, although some interested libraries in applying this standard began to work locally. When RDA Toolkit appeared, some Chilean libraries subscribed to it; however, they continued working isolatedly, without studying the new regulations or the tools as a national group, which lead to a different level of use and application of RDA in every library that decided its implementation. Nevertheless the above mentioned, this document will review the experience developed by the National Congress of Chile Library (BCN), which as of august 2012, generated a project to implement the RDA cataloging model, both in bibliographic records and in authority records. Likewise, the case of the Library System of the University of Concepción, which in 2014 also decided to apply RDA in all its bibliographic records, and a year later in its authority records, will be reviewed. Additionally, other cases in which it has been decided to implement the RDA gradually, in a hybrid way (mix of RCAA2 and RDA), will be approached. Finally, some activities being carried out today in relation to the RDA will be mentioned, as is the case of the training offered by the National Congress of Chile Library to librarians from other institutions of the country, besides the participation in IFLA on the subject of RDA, and future projects related to the training continuation, and the study of the new FRBR-LRM model and the BIBFRAME format. 


\section{Introducción}

En Chile, la implementación de la norma RDA (Recursos, Descripción y Acceso) ha sido particularmente desafiante, puesto que comenzó con mucho entusiasmo por parte de la comunidad bibliotecaria nacional, con la Biblioteca Nacional de Chile (BN) como líder en este cambio, pero después de unos años y de algunos cambios en las instituciones públicas (principalmente en la Dirección de Bibliotecas, Archivos y Museos, DIBAM), este entusiasmo colectivo fue decayendo, quedando sólo en algunas instituciones, sobre todo después de que la BN dejara el liderazgo del Grupo de Interés RDA Chile (GIRCH).

Posteriormente a la suspensión de las actividades del GIRCH, que ocurrió en el año 2014 (Biblioteca Nacional de Chile, 2014), algunas bibliotecas siguieron estudiando y aplicando las RDA en sus registros bibliográficos y de autoridades. Es así como la Biblioteca del Congreso Nacional de Chile continuó trabajando con capacitaciones internas, traduciendo las RDA Toolkit al español y aplicándolas principalmente a los registros de autoridad de personas y a los registros bibliográficos. Asimismo, el Sistema de Bibliotecas de la Universidad de Concepción y la Biblioteca de la Universidad Alberto Hurtado continuaron estudiando las RDA, aplicándolas en diferentes niveles, en sus respectivos registros bibliográficos y de autoridades.

Más adelante se fueron sumando otras instituciones interesadas en continuar, o iniciar, el estudio de las RDA y su aplicación, por lo que se comenzó a incluir el tema de esta normativa en talleres, charlas y seminarios a nivel nacional. Debido a esto, en el año 2015 se realizaron dos actividades en la Biblioteca Central de la Universidad de Concepción; en mayo se realizó el "Seminario de nombres de personas y de entidades corporativas: registros de autoridad RDA/FRAD/MARC21", impartido por el Maestro Ageo García, y en noviembre el "Curso: Creación de registros bibliográficos bajo la norma RDA/FRBR/MARC21", impartido por las bibliotecarias de la propia institución. En el mes de diciembre del mismo año se realizó la "Capacitación en el estándar de catalogación RDA: teoría y práctica", en las dependencias del Congreso Nacional en Santiago y en la Biblioteca Nacional. Dicha actividad fue impartida por dos bibliotecarias del Departamento de Producción de Recursos de Información de la Biblioteca del Congreso Nacional de Chile. Durante el año 2016 se ha continuado con las charlas sobre las RDA por parte del Colegio de Bibliotecarios de Chile y por un instituto de formación para bibliotecarios.

\section{Grupo de Interés RDA Chile}

El Grupo de Interés RDA Chile (GIRCH) se formó en el año 2010 con el objetivo de "Estudiar los nuevos estándares para la descripción y acceso de los recursos de información" (Biblioteca Nacional de Chile, 2014) y se propusieron como actividades el "Estudio y futura implementación de las RDA" (Biblioteca Nacional de Chile, 2014). Para lograr ese fin se reunió un grupo de bibliotecarios de distintas instituciones del país, lo que ha quedado plasmado como testimonio en la página web que se creó bajo el auspicio de la Biblioteca Nacional de Chile (Biblioteca Nacional de Chile, 2014), en donde también se depositaban los documentos de este grupo. Las instituciones participantes fueron:

Biblioteca del Congreso Nacional de Chile

Biblioteca Nacional de Chile

Instituto Carlos Casanueva

Pontificia Universidad Católica de Chile

Universidad Alberto Hurtado 
Universidad de Chile Universidad de Concepción

Universidad de Los Andes

Universidad de Santiago

Universidad del Bío-Bío

Universidad Católica del Norte

Biblioteca del Poder Judicial

En el acta de la reunión del 26 de agosto de 2010, quedó plasmado el registro del comienzo de las actividades del GIRCH, donde se declara que el objetivo general de esta reunión fue "dar inicio a las actividades del GIRCH, determinar los ejes de trabajo del Grupo y consensuar la metodología de trabajo" (Grupo de Interés RDA-Chile, 2010a). Entre los acuerdos de esa primera reunión se menciona que la "metodología de trabajo será en un $80 \%$ en línea, y que consistirá en que cada institución participante... tendrá su espacio en el sitio biblioestandares.bn.cl para enviar información y subir documentos preliminares..." (Grupo de Interés RDA-Chile, 2010a). Con este acuerdo se buscaba integrar a los colegas bibliotecarios de las distintas regiones de Chile, así como utilizar las herramientas tecnológicas disponibles como metodología de trabajo para un grupo que se esperaba que creciera en número de participantes y en documentación aportada por cada uno de ellos. Asimismo, en esta reunión se acuerda que los participantes del Grupo ingresarán al sitio RDA Toolkit para conocer esta nueva herramienta y poder trabajar en base a ella en las próximas reuniones.

En su corta vida, el GIRCH realizó variadas actividades, las cuales son mencionadas en la ponencia presentada por Erika Catillo y Katia Venegas (Venegas Foncea y Castillo Sáez, 2013) en las IV Jornadas "Temas Actuales en Bibliotecología", el 1 de Noviembre de 2013, siendo enlistadas las siguientes:

- Traducción de la tabla de contenidos de las RDA, realizada por la Sra. Patricia Lillo en octubre de 2010.

- Videoconferencia con la experta, y propiciadora de las RDA, de la Library of Congress (LC), Sra. Barbara Tillett, realizada a fines de 2010.

- Capacitación teórica sobre "RDA y su relación con otros estándares," dictada por el invitado internacional, Sr. Gerardo Salta, en la Sala América de la BN, el 8 de noviembre de 2012.

- Colaboración en la traducción de los materiales de capacitación sobre las "RDA para registros bibliográficos", elaborados por la Library of Congress (LC), enero a marzo de 2013.

- Participación en las charlas "Nuevos métodos de indexación y catalogación de las RDA," organizadas por el Colegio de Bibliotecarios, en mayo de 2013, con las ponencias: "RDA: Factor de impacto" por la Sra. Patricia Ortiz. "RDA: Descripción y Acceso al Recurso: un Código de Catalogación para el entorno digital", por la Sra. Erika Castillo Sáez.

- Documento "Abreviaturas en desuso en Registros Bibliográficos y campo MARC asociado," compilado por Alejandra Muñoz y Katia Venegas y coordinado por Erika Castillo, abril de 2013.

- Capacitaciones sobre las "Normas de Catalogación RDA para bibliográficos," realizadas los días miércoles 21 y 28 de agosto, así como los miércoles 4 y 25 de septiembre del 
año 2013, en la Biblioteca del Congreso Nacional y transmitidas vía streaming.

- Actualización permanente del espacio web para interactuar y difundir los documentos generados por el Grupo y complementar con bibliografía, disponible en http://biblioestandares.bn.cl/

En ese mismo trabajo, las autoras declaran que se espera implementar las RDA paulatinamente, después que se concluyan las capacitaciones que se estaban llevando a cabo, así como una vez que se contara con las RDA traducidas al español.

Además de las reuniones del Grupo central, se efectuaron otras reuniones de subgrupos que nacieron a partir de la iniciativa del GIRCH. Uno de ellos fue el Subgrupo de Interés RDA-Chile, Puntos de Acceso, el cual dio comienzo a sus actividades el día 22 de julio de 2011, declarando como su objetivo general: "dar la partida oficial al subgrupo... quien estudiará la Sección 2: Obras y Expresiones, y la Sección 3: Personas, familias y entidades corporativas, de la RDA de acuerdo a la presentación del Mapeo de Autoridades RDA a MARC..." (Grupo de Interés RDAChile, 2011). En esa primera reunión, se planteó una metodología de estudio y se acordó comenzar con el estudio de la Sección 2 de las RDA. Anteriormente, el día 5 de agosto de 2010, se había llevado a cabo una Reunión de Catalogadores, reunión ampliada en que se buscaba "compartir experiencias y problemas relacionados con la descripción documental y el control de autoridades" (Grupo de Interés RDA-Chile, 2010b), donde además se propuso formar grupos de trabajo divididos por los diferentes formatos para hacer más eficiente el estudio de esta norma.

El día 4 de julio del año 2014, a través de un comunicado en el sitio web de Normas y Estándares Bibliográficos de la BN, se informa la suspensión de actividades del GIRCH debido a una reestructuración interna del Departamento de Procesos Técnicos y de la Sección de Catalogación de la Biblioteca Nacional de Chile. En este comunicado se declara que la BN deja el liderazgo del Grupo, dando la opción para que otra institución participante asuma esa responsabilidad (Biblioteca Nacional, 2014). Luego de esa notificación se detienen las actividades del GIRCH, y ninguna de las otras instituciones participantes toma el liderazgo del Grupo; aunque algunas de ellas siguieron avanzando y estudiando las RDA de manera individual (o interna), otras no continuaron con este tema, esperando que la Biblioteca Nacional, u otra, retome el liderazgo del GIRCH.

\section{Experiencias de implementación de RDA en bibliotecas chilenas}

En el artículo "La RDA ¿Mito o realidad?: Panorámica de la catalogación en Chile" (Vargas Rodríguez y Zamorano Pérez, 2012), se hace un estudio sobre el nivel de conocimiento y de la implementación de las RDA en el ámbito nacional. En primer lugar se les preguntó a los catalogadores chilenos sobre cómo estaban desarrollando el estudio de las RDA, siendo los resultados los siguientes: el 39\% declara que las estudiaba de forma individual, el $23 \%$ en un grupo de trabajo a nivel nacional, el $15 \%$ en un grupo de trabajo interno y el $23 \%$ por medio de otros. Como conclusión de esta pregunta se menciona que las "universidades privadas tienen un grupo de estudio de la RDA establecido, y las universidades tradicionales también tienen uno, el cual es comandado por la Biblioteca Nacional" (Vargas Rodríguez y Zamorano Pérez, 2012). Asimismo, en el estudio se consultó sobre la posibilidad de implementar las RDA por parte de los sistemas integrados de información (ILS) que utilizaba cada institución, y solo 
el $16 \%$ declaró que se podría implementar con la tecnología usada en ese momento.

En el estudio revisado se habla de la implementación de las RDA en tiempo futuro, ya que durante el año 2012 se estaba trabajando precisamente en ese proceso de estudio de la norma, pensando en una futura implementación, revisando y analizando las ventajas y desventajas de ser los pioneros en ponerla en práctica, o considerando si era mejor esperar a que se realizara una implementación a nivel nacional, liderada por la Biblioteca Nacional.

En los años posteriores a este estudio, las bibliotecas chilenas, especialmente las de algunas universidades, han continuado con el estudio sistemático de las RDA y con su implementación, ya sea parcial o completa. Entre los casos de aplicación parcial de la norma, se puede citar el caso de la Biblioteca de la Universidad Alberto Hurtado, $\underline{1}$ que fue parte del GIRCH y ha continuado con el trabajo de aplicación de las RDA. En este caso se implementó de una manera "híbrida", puesto que sus registros conviven con los registros creados bajo las RCAA2 y el trabajo que se ha efectuado ha sido incorporar algunos campos MARC pertinentes a la nueva norma, como por ejemplo los campos 336-337-338.

Otro caso de implementación parcial de las RDA se encuentra en la Biblioteca Nacional de Chile, que, luego de liderar el GIRCH, no había continuado con el tema de la implementación de esta norma en sus registros. Sin embargo, en el año 2015 comenzó a trabajar con la aplicación de las RDA en un grupo de registros, con el objetivo de estudiar y evaluar el tiempo que tomaba crear registros utilizando los "nuevos" campos MARC que consideran las RDA. Para realizar este ejercicio se decidió trabajar con los registros de literatura chilena en los cuales utilizan los campos MARC 264, 336-337-338.

En el caso del Sistema de Bibliotecas de la Universidad de Chile, $\underline{\underline{2}}$ solo se han creado algunos registros de autoridad utilizando las RDA, debido a que se necesitaban ciertos datos que se podían generar utilizando los campos MARC/RDA de autoridad personal. No obstante, más allá de este caso puntual, no se ha incorporado el trabajado con la norma, aunque los miembros de la Biblioteca han estado asistiendo a capacitaciones y charlas sobre las RDA.

Entre los casos de implementación completa o total de las RDA, cabe destacar el trabajo realizado por las bibliotecarias de la Biblioteca Central de la Universidad de Concepción, quienes también fueron parte del $\mathrm{GIRCH}$, al continuar estudiando la norma y desarrollando pruebas de aplicación, en conjunto con el Área de Informática de dicha institución. En este caso, las profesionales desarrollaron grupos de estudio interno y trabajaron en forma paralela a las reuniones del GIRCH, de lo cual surgió el "Proyecto de Implementación RDA en Bibliotecas UdeC" (Serrano, 2014), que se ejecutó en tres etapas: investigación, estudio e implementación. En el año 2014 se transfirió el 100\% de la base de datos de registros bibliográficos a RDA y durante el año 2015 estuvieron trabajando con la transferencia de la base de datos de registros de autoridades. Paralelamente a la implementación de la norma, la Biblioteca de la Universidad de Concepción realizó dos capacitaciones sobre RDA durante el año 2015: en mayo el "Seminario de nombres de personas y de entidades corporativas: registros de autoridad RDA/FRAD/MARC21", impartido por el Maestro Ageo García, y en noviembre el curso "Creación de registros bibliográficos bajo la norma RDA/FRBR/MARC21", impartido por las bibliotecarias de la propia institución. Adicionalmente, entre el 10 y el 13 de mayo del año 2016 se realizó el "Taller de Capacitación NACO: creación de registros de autoridades con pautas RDA/FRAD/MARC21", también impartido por el señor Ageo García. De esta última actividad surgió el Proyecto Chimenea NACO Chile, el primero en el Cono Sur, el cual está conformado por siete instituciones: 
Biblioteca del Congreso Nacional de Chile Universidad Federico Santa María de Valparaíso

Universidad Católica del Maule

Universidad Católica de la Santísima Concepción

Universidad de la Frontera

Universidad San Sebastián

Universidad de Concepción (coordinador)

Durante el transcurso del año 2016, las instituciones mencionadas han seguido trabajando, enviando registros a las bibliotecarias de la Universidad de Concepción, quienes son las coordinadoras del proyecto y las encargadas de subir los registros a la base de datos de OCLC, para que finalmente sean visibles desde el sitio de Autoridades de la Biblioteca del Congreso de Estados Unidos.

Estos son solo algunos casos en que se han implementado, o se han estado implementando los cambios que conllevan las RDA en las bibliotecas chilenas. Cabe destacar que existe un gran interés en las capacitaciones y charlas que se realizan sobre las RDA, lo cual se expresa en el gran número de participantes en las capacitaciones mencionadas y en todos los espacios que se dan para conversar y compartir experiencias respecto a la implementación de las RDA en las diferentes bibliotecas a lo largo de Chile.

\section{Experiencia de implementación de RDA en la Biblioteca del Congreso Nacional de Chile}

La Biblioteca del Congreso Nacional de Chile (BCN), a través de la labor desarrollada por los bibliotecarios que trabajan en el Departamento de Producción de Recursos de Información, ha sido pionera en el uso de la norma RDA en la catalogación de registros bibliográficos y de autoridades. La BCN fue integrante del GIRCH desde el comienzo de sus funciones y participó en forma activa de las reuniones de este grupo, incluso una de las capacitaciones más relevantes (transmitida vía streaming para todo Chile) se realizó en las instalaciones de su sede Huérfanos, en Santiago de Chile.

Cuando apareció el RDA Toolkit, la Biblioteca del Congreso se suscribió a esta herramienta y se comenzó a estudiar de manera sistemática esta nueva norma de catalogación. Paralelamente al trabajo y participación en el GIRCH, en la BCN se planificó una serie de capacitaciones internas bajo el proyecto "Implementación de modelo de catalogación RDA en la BCN", en agosto de 2012. Dentro de este proyecto, se desarrolló un plan de capacitación destinado a bibliotecarios y técnicos bibliotecarios del Departamento de Producción de Recursos de Información, el cual se llevó a cabo en sesiones semanales de 2 horas de duración, entre los meses de agosto y diciembre del año 2012. El objetivo de estas capacitaciones fue: "Conocer la herramienta en línea RDA Toolkit y su principal componente las RDA: Recursos, Descripción y Acceso" (Muñoz y Quiroz, 2015), a través del entrenamiento en el uso del kit de herramientas para acceder y aplicar las nuevas normas, así como aprender a buscar, navegar, personalizar el kit y encontrar ayuda y recursos adicionales. Además, se elaboró una propuesta de trabajo para realizar los cambios que implicaban las RDA y se trabajó con ejemplos reales de registros bibliográficos.

El plan de trabajo para la implementación de las RDA en la Biblioteca del Congreso se dividió en dos fases: 
Fase 1: Implementación de nuevos campos del formato MARC21 / RDA de datos bibliográficos (año 2012 y 2013).

Fase 2: Trabajo con base de datos de autoridades (año 2013 y 2014).

Durante la Fase 1 se hicieron una serie de requerimientos de cambios globales en la base de datos de registros bibliográficos en el sistema Horizonte, como ser el desarrollo de las abreviaturas utilizadas bajo el estándar RCAA2 y la incorporación de nuevos campos y subcampos (Biblioteca del Congreso Nacional de Chile, 2013b). Durante la Fase 2 se generó como meta del Departamento de Producción (Biblioteca del Congreso Nacional de Chile, 2013a), la implementación de las RDA a un total de 344 registros de autoridades de senadores y diputados con el rol de autores, como una forma de aprendizaje a través del nuevo estándar de tratamiento de autoridades personales. Posteriormente a lo realizado en ambas fases de trabajo, se ha seguido con la aplicación de las RDA, tanto en registros bibliográficos como de autoridades.

Los principales cambios incorporados con la implementación de RDA fueron:

Registros bibliográficos:

- Campos fijos:

000 Líder 18 con valor "i".

- Campos variables:

Campo 040 \$e contiene el código "rda";

Campo 245 \$c mención de responsabilidad, incorporación de todos los autores en el caso de una publicación chilena y reemplazo de abreviatura et. al por [y otros...] ;

Se incorpora el uso del campo $264 ; \mathrm{y}$,

Se incluyen en la plantilla de trabajo los campos 336-337-338.

Registros de autoridad:

- Campos fijos:

000 Líder 18 con valor "i".

$008 / 10=z$ (otro)

- Campos variables:

Campo 040 \$e contiene el código "rda";

Campo 046, Códigos especiales para fechas;

Campo 368, Otros atributos de la entidad corporativa;

Campo 370, Lugar asociado;

Campo 371, Dirección;

Campo 372, Actividad;

Campo 373, Grupo asociado;

Campo 374, Profesión u ocupación; 
Campo 375, Género;

Campo 377, Lengua asociada;

Campo 378, Nombre completo de la Persona

En relación a las capacitaciones externas, la Biblioteca del Congreso realizó un taller de Capacitación en RDA en octubre de 2012 y en agosto y septiembre de 2013, en el marco de las actividades del GIRCH. El 17 y 18 de diciembre del año 2015 se realizó la "Capacitación en el estándar de Catalogación RDA: teoría y práctica", en la Sala de Lectura de la Cámara de Diputados, en la sede del Congreso Nacional en Santiago y en el Salón Bicentenario de la Biblioteca Nacional, la cual contó con la participación de bibliotecarios de la Biblioteca Nacional, el Sistema de Bibliotecas Públicas, la Biblioteca de la Corte Suprema, la Pontificia Universidad Católica, la Universidad de Santiago, el Museo Nacional de Bellas Artes y la Biblioteca del Congreso. En esta capacitación la BCN compartió su experiencia respecto a la implementación de RDA, mostrándose los principales cambios incorporados y desarrollándose ejercicios prácticos.

Por otra parte, los bibliotecarios de la BCN también han participado en diversas capacitaciones, charlas y talleres relacionados con la norma RDA, desde la aparición de ésta en el año 2009, a través del proceso que significó la participación en el $\mathrm{GIRCH}$, las experiencias que se han presentado principalmente en los congresos anuales organizados por el Colegio de Bibliotecarios de Chile y por el Servicio de Información y Bibliotecas de la Universidad de Chile, así como por las capacitaciones realizadas en la Biblioteca Central de la Universidad de Concepción.

Finalmente, cabe destacar que en la reunión satélite "RDA in the Wider World", en el marco del Congreso Mundial de Bibliotecas e Información 2016 de la IFLA realizado en Dublín, Ohio, USA, se llevó a cabo la presentación de la ponencia "Impact of RDA in Chile: complex scenario and adaptation to change" (Quiroz, 2016). Asimismo, en el segundo semestre del año 2016, se comenzó con el estudio de BIBFRAME y el Open Linked Data, como opciones para transformar los registros en formato MARC21, de acuerdo a los estándares de la web semántica y a los requerimientos de los nuevos usuarios de la información.

\section{Reflexiones finales}

El estudio sobre la implementación de las RDA en Chile comenzó en el momento de la aparición de la herramienta en línea RDA Toolkit. Dicho estudio se trabajó a nivel nacional en el Grupo de Interés RDA-Chile, liderado por la Biblioteca Nacional, con representación de diversas instituciones a lo largo de Chile.

Una vez que el GIRCH dejó de funcionar, las instituciones que formaban parte de éste se separan y continúan trabajando de manera individual, algunas de ellas siguiendo con el estudio para la implementación de las RDA en sus registros bibliográficos y de autoridades, como es el caso de la Biblioteca de la Universidad Alberto Hurtado, el Sistema de Bibliotecas de la Universidad de Concepción y la Biblioteca del Congreso Nacional. Algunas otras instituciones han comenzado también con la implementación a través de proyectos o muestras de registros de sus respectivos sistemas integrados.

A través de diversas charlas, seminarios y presentaciones a nivel nacional, realizadas durante los años 2014 y 2015, se constata el resurgimiento del interés por implementar las RDA en las distintas bibliotecas a lo largo de Chile. Este interés se demuestra en la asistencia a los talleres 
mencionados en el trabajo, los cuales han tenido una alta participación de bibliotecarios de las distintos tipos de bibliotecas, ya sean universitarias, públicas o especializadas.

La Biblioteca del Congreso Nacional de Chile ha tenido una activa participación en el desarrollo del estudio de las RDA en Chile y en su implementación, tanto por su participación en el GIRCH, como por auspiciar y desarrollar capacitaciones internas y externas. La experiencia de la Biblioteca del Congreso de Chile también ha sido presentada y difundida a nivel internacional. Por otro lado, se han empezado proyectos para la adopción de nuevos estándares relacionados con RDA como BIBFRAME y Open Linked Data.

Finalmente, es importante mencionar que la Biblioteca del Congreso Nacional de Chile ha sido seleccionada a partir de 2017 como la Institución Nacional que representará a América Latina y el Caribe en el Consejo de las RDA (RDA Board).

\section{Notas}

1 Datos recolectados por correo electrónico, 8 de Marzo de 2016.

$\underline{2}$ Datos recolectados por correo electrónico, 13 de Abril de 2016.

\section{Referencias bibliográficas}

Biblioteca del Congreso Nacional de Chile, Sección Producción de Fuentes Referenciales. (2013a). Meta No. 1 Generar aprendizaje a través de los nuevos estándares de tratamiento de autoridades personales aplicando RDA y FRAD a 164 registros de senadores y diputados autores. Santiago, Chile: Biblioteca del Congreso Nacional de Chile (no publicado).

Biblioteca del Congreso Nacional de Chile, Sección Producción de Fuentes Referenciales. (2013b). Meta No. 6. Adecuar la base de datos Horizonte al nuevo estándar RDA, para obtener registros bibliográficos potenciados con la nueva norma de catalogación. Santiago, Chile: Biblioteca del Congreso Nacional de Chile (no publicado).

Biblioteca Nacional de Chile. (2014). Normas y Estándares Bibliográficos. Santiago, Chile. Recuperado de http://biblioestandares.bn.cl/

Grupo de Interés RDA-Chile. (2010a). Acta de Reunión del 26 de agosto de 2010. Santiago, Chile (no publicado).

Grupo de Interés RDA-Chile (2010b). Reunión de Catalogadores. Acta No 1 del 5 de Agosto de 2010. Santiago, Chile (no publicado).

Grupo de Interés RDA-Chile. Subgrupo de Interés RDA-Chile, Puntos de Acceso. (2011). Acta de Reunión del 22 de julio de 2011. Santiago, Chile (no publicado).

Muñoz, A. y Quiroz, Á. (2015). Capacitación en el estándar de catalogación RDA: teoría y práctica. Santiago, Chile: Biblioteca del Congreso Nacional de Chile (no publicado).

Quiroz, Á. (2016). Impact of RDA in Chile: complex scenario and adaptation to change. Recuperado https://www.oclc.org/content/dam/oclc/events/2016/IFLA2016/presentations/Impact-of-RDAin-Chile.pdf

Serrano, M. T.. (2014). Sistema de Bibliotecas Universidad de Concepción. Experiencia de 
implementación RDA. Trabajo presentado en XVIII Conferencia Internacional de Bibliotecología 2014. Santiago, Chile. Recuperado de http://bibliotecarios.cl/descargas/2015/03/07experiencia-de-implementacion-rda.pdf

Vargas Rodríguez, C. y Zamorano Pérez, A. (2012). La RDA ¿Mito o realidad?: Panorámica de la catalogación en Chile. Serie Bibliotecología y Gestión de Información, 72. Recuperado de http://eprints.rclis.org/16842/

Venegas Foncea, K. y Castillo Sáez, E. L. (2013). La Biblioteca Nacional de Chile y el nuevo código de catalogación RDA. Trabajo presentado en IV Jornada Temas Actuales en Bibliotecología. Mar del Plata. Recuperado de http://eprints.rclis.org/20609/ 